Leonhard, Tobias

\title{
Fachlichkeit in zwei Praxen zur Geltung bringen. Beiträge der Berufspraktischen Studien zur fachlichen Professionalisierung von Lehrpersonen des Zyklus 1
}

Bachmann, Sara [Hrsg.]; Bertschy, Franziska [Hrsg.]; Künzli David, Christine [Hrsg.]; Leonhard, Tobias [Hrsg.]; Peyer, Ruth [Hrsg.]: Die Bildung der Generalistinnen und Generalisten. Perspektiven auf Fachlichkeit im Studium zur Lehrperson für Kindergarten und Primarschule. Festschrift für Frau Prof. Dr. Charlotte Müller. Bad Heilbrunn : Verlag Julius Klinkhardt 2021, S. 194-209. - (Studien zur Professionsforschung und Lehrerbildung)

Quellenangabe/ Reference:

Leonhard, Tobias: Fachlichkeit in zwei Praxen zur Geltung bringen. Beiträge der Berufspraktischen Studien zur fachlichen Professionalisierung von Lehrpersonen des Zyklus 1 - In: Bachmann, Sara [Hrsg.]; Bertschy, Franziska [Hrsg.]; Künzli David, Christine [Hrsg.]; Leonhard, Tobias [Hrsg.]; Peyer, Ruth [Hrsg.]: Die Bildung der Generalistinnen und Generalisten. Perspektiven auf Fachlichkeit im Studium zur Lehrperson für Kindergarten und Primarschule. Festschrift für Frau Prof. Dr. Charlotte Müller. Bad Heilbrunn : Verlag Julius Klinkhardt 2021, S. 194-209 - URN: urn:nbn:de:0111-pedocs-222119 - DOI: 10.25656/01:22211

https://nbn-resolving.org/urn:nbn:de:0111-pedocs-222119 https://doi.org/10.25656/01:22211

in Kooperation mit / in cooperation with:

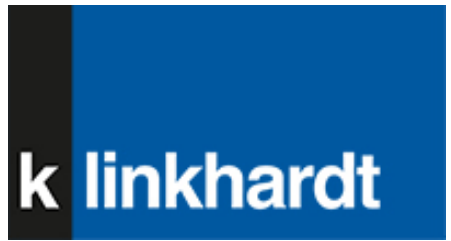

http://www.klinkhardt.de Nutzungsbedingungen

Dieses Dokument steht unter folgender Creative Commons-Lizenz: http://creativecommons.org/licenses/by-nc-sa/4.0/deed.de - Sie dürfen das Werk bzw. den Inhalt unter folgenden Bedingungen vervielfältigen, verbreiten und öffentlich zugänglich machen sowie Abwandlungen und Bearbeitungen des Werkes bzw. Inhaltes anfertigen: Sie müssen den Namen des Autors/Rechteinhabers in der von ihm festgelegten Weise nennen. Dieses Werk bzw. der Inhalt darf nicht für kommerzielle Žwecke verwendet werden. Die neu entstandenen Werke bzw. Inhalte dürfen nur unter Verwendung von Lizenzbedingungen weitergegeben werden, die mit denen dieses Lizenzvertrages identisch oder vergleichbar sind.

Mit der Verwendung dieses Dokuments erkennen Sie die Nutzungsbedingungen an.

\section{Terms of use}

This document is published under following Creative Commons-License: http://creativecommons.org/licenses/by-nc-sa/4.0/deed.en - You may copy, distribute and transmit, adapt or exhibit the work in the public and alter, transform or change this work as long as you attribute the work in the manner specified by the author or licensor. You are not allowed to make commercial use of the work. If you alter, transform, or change this work in any way, you may distribute the resulting work only under this or a comparable license.

By using this particular document, you accept the above-stated conditions of use.

\section{Kontakt / Contact:}

\section{DeDOCS}

DIPF | Leibniz-Institut für Bildungsforschung und Bildungsinformation Informationszentrum (IZ) Bildung

E-Mail: pedocs@dipf.de

Internet: www.pedocs.de

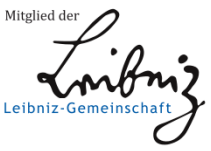


Studien zur Professionsfiorschung

und Lehrerbildung)

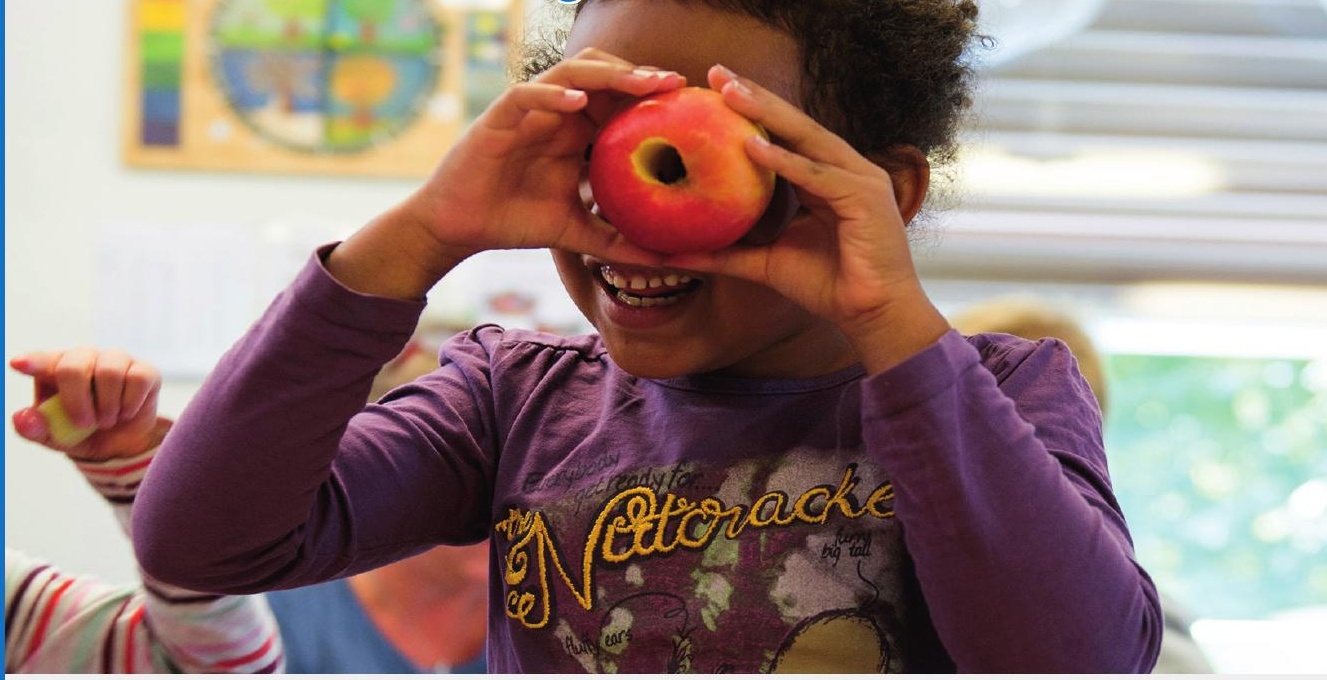

Sara Bachmann / Franziska Bertschy Christine Künzli David / Tobias Leonhard Ruth Peyer

(Hrsg.)

\section{Die Bildung der Generalistinnen und Generalisten}

Perspektiven auf Fachlichkeit im Studium zur Lehrperson für Kindergarten und Primarschule 
Bachmann / Bertschy / Künzli David / Leonhard / Peyer Die Bildung der Generalistinnen und Generalisten 


\section{Studien zur Professionsforschung und Lehrerbildung}

Herausgegeben von

Till-Sebastian Idel, Manuela Keller-Schneider, Katharina Kunze und Christian Reintjes 


\section{Sara Bachmann \\ Franziska Bertschy \\ Christine Künzli David \\ Tobias Leonhard \\ Ruth Peyer \\ (Hrsg.) \\ Die Bildung der Generalistinnen und \\ Generalisten}

Perspektiven auf Fachlichkeit im Studium zur Lehrperson für Kindergarten und Primarschule

Festschrift für Frau Prof. Dr. Charlotte Müller anlässlich ihrer Emeritierung als Leiterin des Instituts Kindergarten-/Unterstufe

Verlag Julius Klinkhardt 
Die Herausgabe des Bandes wurde von der Pädagogischen Hochschule FHNW finanziert.

Dieser Titel wurde in das Programm des Verlages mittels eines Peer-Review-Verfahrens aufgenommen. Für weitere Informationen siehe www.klinkhardt.de.

Bibliografische Information der Deutschen Nationalbibliothek Die Deutsche Nationalbibliothek verzeichnet diese Publikation in der Deutschen Nationalbibliografie; detaillierte bibliografische Daten sind im Internet abrufbar über http://dnb.d-nb.de.

2021.ig. () by Julius Klinkhardt.

Foto Umschlagseite 1: (c) Theo Gamper, Solothurn/Tobias Leonhard.

Druck und Bindung: AZ Druck und Datentechnik, Kempten.

Printed in Germany 2021.

Gedruckt auf chlorfrei gebleichtem alterungsbeständigem Papier. 


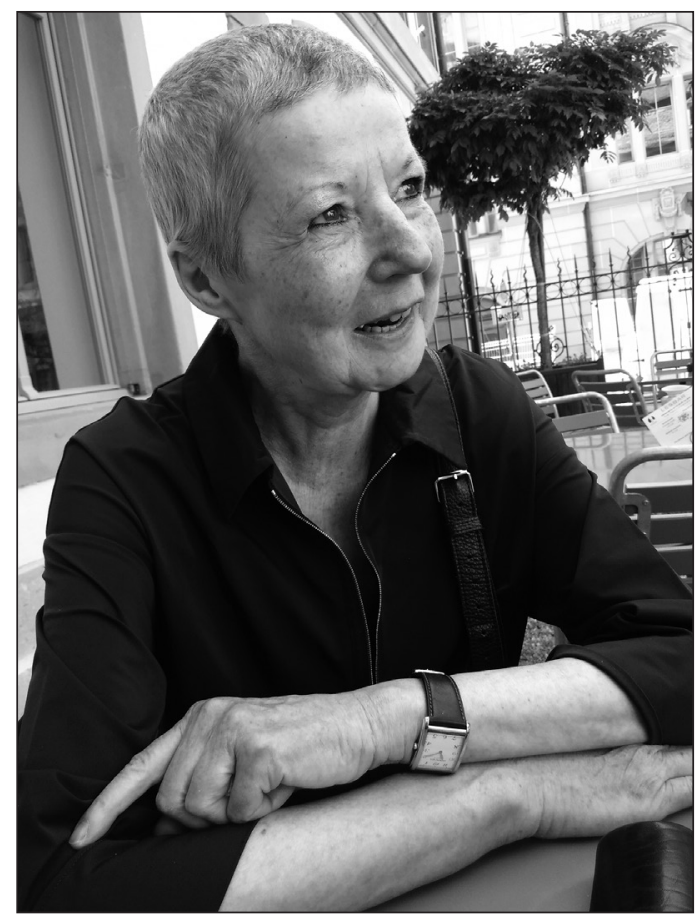

Prof. Dr. Charlotte Müller

Leiterin des Instituts Kindergarten-/Unterstufe

der Pädagogischen Hochschule FHNW von 2009 bis 2021 


\section{Inhaltsverzeichnis}

\section{Vorworte}

Sabina Larcher Klee

,Vielfachlichkeit und Eigensinniges

Sara Bachmann, Franziska Bertschy, Christine Künzli David, Tobias Leonhard und Ruth Peyer „Ich will jetzt endlich mal die Plots sehen!“

\section{Teil 1: Einführung und Problemaufriss}

Sara Bachmann, Franziska Bertschy, Christine Künzli David, Tobias Leonhard und Ruth Peyer

Die Bildung der Generalistinnen und Generalisten

Einleitung, Problemaufriss und Fragehorizont

Teil 2: Fachliche Perspektiven auf generalistische Handlungsanforderungen

Barbara Wyss

Flirten mit der Vernunft

Weltzugang und Welterzeugung durch Ästhetische Bildung

Bildnerisches und Technisches Gestalten im Zyklus 1

Elke Gramespacher, Susanne Störch Mehring,

Zita Bucher und Claudia Klostermann

Bewegungsbildung für Kinder:

Für „Generalistinnen“ und „Generalisten“ nicht nur eine sportdidaktische Herausforderung!

Mathilde Gyger

Zugänge zur Welt: sprachlich realisiert und didaktisch reflektiert Sprachliche Bildung im Zyklus 1 
8 Inhaltsverzeichnis

Christine Streit und Thomas Royar

Zahlen, Formen und Beziehungen

Lernen und Lehren von Mathematik im Zyklus 1

Jürg Zurmühle

Musik im Kindergarten und in der Unterstufe der Primarschule

Franziska Bertschy

Vielperspektivität und Perspektivenverbindung

Bildungsprozesse im Sachunterricht ermöglichen

Teil 3: Konzeptionen zur Stiftung einer professionsspezifischen Kultur von Fachlichkeit für den Zyklus 1

Christine Künzli David und Edwin J. de Sterke

Mehr als Fachlichkeit

Transversales Unterrichten als Spezifik einer Didaktik des Zyklus 1

und als verbindendes Konzept im Studiengang für Lehrpersonen

dieser Stufe

Tobias Leonhard

Fachlichkeit in zwei Praxen zur Geltung bringen

Beiträge der Berufspraktischen Studien zur fachlichen

Professionalisierung von Lehrpersonen des Zyklus 1

Anja Blechschmidt und Leticia Venâncio

Im Team für ALLE Kinder in der inklusiven Bildung unterwegs

\section{Nachwort}

Tobias Leonhard im Gespräch mit Charlotte Müller

Dem Eigensinn Raum geben

Annotationen zu Bildung in Kindergarten, Schule und Hochschule

Autorinnen- und Autorenverzeichnis 
194

\section{Tobias Leonhard}

\section{Fachlichkeit in zwei Praxen zur Geltung bringen Beiträge der Berufspraktischen Studien zur fachlichen Professionalisierung von Lehrpersonen des Zyklus 1}

\section{Einleitung}

Schul- und Berufspraktische Studien ${ }^{1}$ bilden seit einigen Jahren neben den Fachwissenschaften und deren Didaktiken sowie dem erziehungswissenschaftlichen Studium einen eigenständigen akademischen Studienbereich, der sich gleichwohl noch traditionellen Ansprüchen und narrativen Rahmungen ausgesetzt sieht. Die in diesem Sammelband fokussierte Frage, wie die fachliche Bildung von Lehrpersonen des Zyklus 1 angesichts ,nicht fachlicher' Unterrichtsanlässe - präziser: angesichts von Unterrichtsanlässen, die nicht der Logik einzelner Fachbereiche, z.B. als Musik- oder Mathematikunterricht, folgen - zu denken und in einer Konzeption des Studiengangs zu gestalten ist, wird nachfolgend hinsichtlich des möglichen Beitrags der Berufspraktischen Studien diskutiert.

Den Ausgangspunkt des Beitrags bildet im folgenden zweiten Abschnitt zunächst die Darstellung grundlegender konzeptioneller Überlegungen zu einem eigenständigen Studienbereich Berufspraktischer Studien. Ausgehend von der Kritik an traditionellen Vorstellungen der Berufspraktischen Studien als ,Anwendungsfeld zur Verbindung von Theorie und Praxis'skizziere ich eine Alternative, die es möglich macht, die Lehrer*innenbildung generell und die Berufspraktischen Studien im Besonderen systematisch und konzeptionell produktiv(er) zu fassen.

Die Fokussierung auf die zentrale Frage des Bandes nach der fachlichen Bildung von Lehrpersonen des Zyklus 1 und den generalistischen Erwartungen an diesen Personenkreis führt im dritten Abschnitt zur Darstellung der derzeit gültigen Gestaltungsprinzipien des Studienbereiches, die auf mehreren Ebenen bezüglich ihrer Chancen und Grenzen ausgearbeitet werden. Hier wird auch untersucht, wie unter diesen Gestaltungsprinzipien die zweifelsfrei auch an Lehrer*innen des Zyklus 1 zu stellenden fachlichen Ansprüche im Studienbereich der Berufspraktischen Studien manifest werden, welchen Anfechtungen sie, im Angesicht der

1 Im Folgenden wird der Schweizer Terminus „Berufspraktische Studien“ verwendet, der an unserer Hochschule auch die berufsbezogenen Studien schulischer Heilpädagoginnen und Heilpädagogen sowie Logopädinnen und Logopäden beinhaltet. Für die Studiengänge zum Lehrberuf ist ausserhalb der Schweiz für diesen Studienbereich der Begriff „Schulpraktische Studien“ oder „Pädagogisch-praktische Studien“ geläufiger. Die Grossschreibung kennzeichnet die Eigenständigkeit. 
zielstufenspezifischen beruflichen Praxis' ausgesetzt sind und mit welchen institutionellen Herausforderungen der Studienbereich operiert. Im abschliessenden vierten Abschnitt wird gedankenexperimentell geprüft, welche Potenziale, aber auch welche Limitationen und Friktionen eine konsequente Ausrichtung auf die Leitfigur des Transversalen Unterrichtens, wie Künzli David und de Sterke sie in ihrem Beitrag skizzieren, für die Berufspraktischen Studien bereithielte.

\section{Berufspraktische Studien als eigenständiger Studienbereich - konzeptionelle Überlegungen}

Im Versuch, Berufspraktische Studien als eigenständigen akademischen Studienbereich zu konturieren (vgl. Košinár/Leineweber/Schmid 2016; Leonhard/ Herzmann/Košinár 2021) sehen sich der Autor und seine Mitstreiter*innen nicht selten mit traditionellen Vorstellungen konfrontiert, die den Studienbereich einerseits zwar als unverzichtbares Element der Lehrer*innenbildung kennzeichnen, ihn andererseits jedoch in einer Anwendungslogik fassen, die wiederum dem traditionellen Narrativ der ,Verbindung von Theorie und Praxis' folgt. Die heuristische Untauglichkeit des Letzteren ist in den vergangenen Jahren mehrfach kritisiert worden (Hackl 2014; Leonhard 2020; Schrittesser/Hofer 2012); gleichwohl bildet die dichotomische, polarisierende und priorisierende Figur, die ,die Praxis' auf Kosten eines eigenständigen Wertes ,der Theorie‘ als das eigentlich Entscheidende rahmt, oft noch den Hintergrund studentischer Kritik oder der Rückmeldungen von Praxislehrpersonen, die z.B. festhalten, die Studierenden seien zwar ,theoretisch fit', hätten aber ,keinen Rucksack praktischer Fähigkeiten und Fertigkeiten`. Gegen diese Common-Sense-Vorstellung gilt es zu argumentieren, will man die als Studienleistungen ${ }^{2}$ (sic!) kreditierten Anteile des Studiums zum Lehrberuf als akademischen Studienbereich konturieren und diesen in einen tragfähigen konzeptionellen Zusammenhang mit den anderen Studienbereichen bringen. Zugleich bildet das Bachelorstudium der einphasigen Lehrer*innenbildung einen formalen Rahmen, der es verunmöglicht, wie in zweiphasigen Systemen die Ansprüche elementarer berufspraktischer Handlungsfähigkeit z.B. an ein Referendariat zu delegieren, wie dies zumindest vor der Einführung der Praxissemester in Deutschland argumentativ möglich war (vgl. Leonhard 2021).

Eine erste zwingende konzeptionelle Konsequenz in dieser Rahmung besteht also in der Anerkennung, dass Berufspraktische Studien sowohl die Möglichkeit grundlegender berufspraktischer Erfahrung und der Einlassung auf die Anfor-

2 Im Studiengang Kindergarten-/Unterstufe der Pädagogischen Hochschule FHNW wird der Studienbereich mit beachtlichen 48 von 180 ECTS-Punkten und damit mit mehr als 25\% des Gesamtworkloads kreditiert. 
derungen und Rationalitäten der beruflichen Praxis im Zyklus 1 als auch den Gewinn einer ersten Handlungssicherheit beinhalten. Die zweite konzeptionelle Konsequenz besteht jedoch darin, diese in der beruflichen Praxis und im Rahmen von Praktika stattfindenden Erfahrungsräume konzeptionell schlüssig als Teil eines akademischen Studiums zu denken und durch spezifische, auf diese Teilnahme an der beruflichen Praxis hin ausgerichtete Hochschulveranstaltungen einzubinden. Hier wie dort, also in der beruflichen Praxis in Kindergarten und Primarschule sowie in der akademischen Praxis der Hochschulen, stellen sich Fragen von Fachlichkeit, die im vorliegenden Beitrag vertieft untersucht werden. Ausgehend vom Vorschlag Bernd Hackls, ,den Umgang mit Theorie selbst als eine spezifische Praxis zu betrachten" (Hackl 2014, 52, Hervorhebung im Original), entstand in den vergangenen Jahren eine alternative Argumentationsfigur, die sich auch im ersten Teil des Bandes bereits abbildet. Lehrer*innenbildung grundsätzlich in mehreren Praxen zu denken, die eine Teilhabe der Studierenden an ebendiesen als Grundvoraussetzung von Professionalisierungsprozessen fasst, etabliert sich zumindest im Fachdiskurs zunehmend als neue Rahmung lehrer*innenbildender Studiengänge (vgl. Leonhard 2018, 2020; Meister/Hericks 2021; Schneider/Cramer 2020; Schrittesser/Hofer 2012).

Der heuristische und konzeptionelle Vorteil liegt auf der Hand: Man kann das Studium an der Hochschule als eigene Praxis neben der traditionell immer nur als beruflich gedachten Praxis positionieren, kann die spezifischen Teilpraxen einer Hochschule differenzieren und die jeweiligen Praktiken sowie deren spezifische Logiken oder Rationalitäten empirisch untersuchen und beschreiben. Man kann auch präzise fassen, was ,Expertise ${ }^{`}$ oder ,Könnerschaft ${ }^{`}$ in den jeweiligen (Teil-)Praxen ausmacht und wie sie erworben wird. Eine solchermassen gewandelte Rahmung speist sich aus praxistheoretischen Überlegungen (vgl. Hillebrandt 2014, 2015; Reckwitz 2003; Schäfer 2016; Schmidt 2012), die als sozial- und kulturwissenschaftliche Theorien in ihrer Fokussierung auf die Praktik(en) als „,kleinste Einheit' des Sozialen“ (Reckwitz 2003, 290) bzw. Praxen als komplexe soziomaterielle Arrangements eine hohe Deutungskraft für eine gesellschaftliche Teilpraxis wie die Lehrer*innenbildung besitzen. Konzeptionell-programmatisch wird die Figur mindestens zweier Praxen in den Berufspraktischen Studien unseres Instituts in zwei Weisen zugespitzt und vereindeutigt: Die Gegenüberstellung der beruflichen Praxis (von Lehrpersonen) und einer Hochschulpraxis, die im Kern sowohl eine (disziplinär organisierte) Wissenschaftspraxis als auch eine fachliche Praxis (als ästhetische Praxis, sprachliche Praxis etc.) darstellt, macht klar beschreibbar, was an welchen Orten des Studiums, zu holen' ist und in welchen Modi Studieren zu denken ist. Sie macht auch klar beschreibbar, was von Mitarbeitenden in den jeweiligen Praxen erwartbar sein muss: Lehrer*innen, die Studierenden in den Praktika begleiten, sind dann in erster Linie als Könner*innen der beruflichen Praxis adressiert, die auf verschiedenen Wegen im Rahmen des 
Praktikums und eines ,Ausbildungsauftrags' Studierende am Ort des Geschehens an ibren spezifschen Praktiken beteiligen, diese zeigen, modellieren, explizit erklären sowie implizit sozialisatorische Wirkungen hinterlassen, einfach, weil sie in der Praktikumssituation relevante Andere und damit Instanzen beruflicher Subjektivierung sind. Von Hochschulmitarbeitenden ist analog zu erwarten, dass sie Könner*innen der Hochschulpraxis sind, also einerseits fachpraktisch, (fach)wissenschaftlich und (hochschul)didaktisch versiert und andererseits zur systematischen Distanzierung von den eigenen fachlichen Rationalitäten in der Lage sind. Das Studium an der Hochschule ist dann als Beteiligung an ebendiesen akademischen Teilpraxen und Praktiken zu konzipieren, die sowohl als explizite Beteiligung (z.B. als Zeigen und Erklären in der Hochschullehre) als auch im Sinne von sozialisatorischen Wirkungen durch die Teilnahme an hochschulspezifischen Praktiken (lesen, diskursive Auseinandersetzung über Geltungsfragen, methodisch geleitete Analyse $^{3}$ ) zu denken ist.

Als argumentativ hilfreiche Figur hat sich dazu auch die neuwegsche Unterscheidung von „Distanz und Einlassung“ (Neuweg 2011) erwiesen, die beschreibbar macht, wie unterschiedlich - auf der Ebene des konkreten Tuns bzw. der Praktiken - ein Studium aussieht. Ob als Einlassung auf die fachliche Praxis des Musizierens als Zentralelement, Ausdrucksmedium und integrierende Chancenstruktur im Zyklus 1 oder als ,reflexive', konkreter: daten- und methodengestützte Distanzierung von der Unmittelbarkeit der Erfahrungen im Praktikum, die zugleich die Einlassung auf die Systematik und argumentative Strenge einer Wissenschaftspraxis erfordert: Das Narrativ mehrerer Praxen erweist sich als erheblich produktiver und deutungsmächtiger für die Verhältnisse der Lehrer*innenbildung als das traditionelle und unlösbare ,Theorie-Praxis-Problem'. Es vermag - wiederum die Einlassung vorausgesetzt - des Weiteren existierende Vorstellungen und Bedürfnisse Studierender nach dem einer impliziten Ausbildungslogik folgenden permanenten ,Praxisbezug ${ }^{64} \mathrm{zu}$ irritieren und damit die notwendige Voraussetzung für eine distanzierte daten- und methodengestützte Analyse zu begünstigen. Eine Modifikation derartiger Common-Sense-Erwartungen scheint dabei umso wahrscheinlicher, je einheitlicher und plausibler eine Hochschule in der Lage ist, sicht- und erfahrbar zu machen, welcher Gewinn aus der Einlassung auf die Wissenschaftspraxis z.B. für die Planung oder das nachträgliche Verstehen von Unterricht entstehen kann.

3 Solche Analysen gegenüber Studierenden gerade nicht als „Forschung“ zu rahmen und dennoch Kernaspekte von Forschung als Wissenschaftspraxis mit Studierenden zu betreiben, reduziert Vorbehalte, die Studierende bisweilen in der Art von „Ich will doch Lehrer*in werden und nicht Forscher*in" artikulieren.

4 Vgl. dazu auch die Untersuchung von Wenzl, Wernet und Kollmer (2018), die den Praxiswunsch Studierender auf der Basis von Interviews als „diffuses Ressentiment“ (Wenzl et al. 2018, 2) gegenüber einem Studium dekonstruieren. 
Fokussiert man die generell für die Rahmung der Lehrer*innenbildung vorgeschlagenen Konzepte auf die Berufspraktischen Studien, wird deutlich, wie sich der Studienbereich zwischen der Hochschulpraxis und der beruflichen Praxis von Lehrpersonen positioniert. Dabei lässt sich auch konkretisieren, welche Zusammenhänge zwischen den verschiedenen Praxen existieren. Ich verdeutliche diese Zusammenhänge an zwei hochschulischen Teilpraxen: zunächst an der derjenigen Teilpraxis, die ich als ,Fachpraxis' bezeichne und bezogen auf den richtigen Ort im Professionalisierungsprozess untersuche, anschliessend an der Teilpraxis, die ich pointiert, Wissenschaftspraxis' nenne.

Mit ,Fachpraxis` ist der Erwerb zumindest elementarer Könner*innenschaft spezifisch fachlicher Operationen gemeint. Wer als Lehrer*in des Zyklus 1 mit Kindern singen, mathematisch denken oder über das gute Leben philosophieren möchte, muss selbst in der Lage sein, diese Operationen gekonnt auszuführen. Zusätzlich - und damit schliesse ich an das Konzept „reflektierter Fachlichkeit“ an (Bonnet 2019, 2020; Meister/Hericks 2021) - gilt es, „[e]rstens eine reflektierte Kenntnis der Inhalte, Methoden und Denkstile der eigenen Fächer, zweitens ein Verständnis typischer Aneignungsprobleme und Differenzen zwischen Fach- und Alltagswissen in den eigenen Fächern, und drittens ein adressaten- und fachgerechtes Konzept fachlicher Bildung [zu entwickeln]" (Bonnet 2019, 174). Es scheint evident, dass alle vier vorgenannten Aspekte kaum ,en passant' im Rahmen der Berufsausübung erworben werden, sondern gerade in den reflexiven Bezugnahmen der expliziten und von unmittelbarem Handlungsdruck entlasteten Thematisierung bedürfen. Damit ist die Hochschule der richtige Ort, an dem fachpraktisches Können und eine reflektierte Fachlichkeit erworben werden können. Diese Praxis als Voraussetzung für den Erwerb der notwendigen Könner*innenschaft ist der beruflichen Praxis von Lehrpersonen zeitlich vorgeordnet.

Das Verhältnis der, Wissenschaftspraxis‘ zur beruflichen Praxis von Lehrpersonen diskutiere ich in einer anderen zeitlichen Dimension, indem ich frage, welcher Gewinn aus der Wissenschaftspraxis für die berufliche Praxis resultieren kann. Hier fokussiere ich nun tatsächlich auf die Berufspraktischen Studien. Studierenden stehen in einem Praktikum zunächst immer wieder (zukunftsbezogen) vor der Aufgabe, Unterricht zu planen, um idealerweise Bildungsprozesse bei möglichst allen Kindern anzustossen. Eine solche Planung gewinnt mit dem Wissen (als Materialisierung systematisierender Überlegungen der Wissenschaftspraxis) und den darin vorgenommenen Unterscheidungen zweifelsohne an Qualität. Ein Konzept zur Differenzierung von Unterricht (vgl. Klafki/Stöcker 1976) kann bei verständiger Auseinandersetzung Dimensionen der Differenzierung sichtbar machen, die alltagsweltlich nicht in den Blick geraten. Die fachlich differenzierte Auseinandersetzung mit den naturwissenschaftlichen Grundlagen sachunterrichtlicher Phänomene wie „Fliegen“ oder "Schwimmen und Sinken“ schafft Beschreibungs- 
kategorien, die notwendige Grundlage einer fachlich adäquaten unterrichtlichen Bearbeitung sind.

Gegenwartsbezogen - also in der konkreten (Mit-)Gestaltung von Unterricht im Rahmen des Praktikums - scheint die Idee einer parallelen Wissenschaftspraxis störend. Dem ist insofern zuzustimmen, als die Einlassung auf die berufliche Praxis und deren kontingente Anforderungen eine gleichzeitige distanzierte und explizierte Beobachtung verunmöglicht; zumindest ist das für Berufseinsteigende anzunehmen, deren Aufmerksamkeit voll vom interaktiven Geschehen beansprucht wird. Gleichwohl erweist sich gerade in diesem Modus, ob vorgängige Wissenschaftspraxis Spuren hinterlassen hat, weil (theoretische) Konzepte, die subjektive Bedeutsamkeit erlangen konnten, nun als inkorporierte Unterscheidungen die interaktionsaufmerksame Wahrnehmung fundieren. Wenn man den Modus interaktiven, situationssensitiven und adaptiven Handelns mit Volpert $(2003,137)$ als „improvisierend-intuitiv“ kennzeichnet, bildet die vorgängige einlassende Befassung mit theoretischen Konzepten (neben Erfahrungen) einen Teil des impliziten Fundus für intuitiv vorgenommene Unterscheidungen als Grundlage spontanen Handelns.

Die dritte Relation ist die vergangenheitsbezogene Befassung von Wissenschaftspraxis mit (kleinen Ausschnitten) der beruflichen Praxis. Hier spielt die Wissenschaft ihr volles Potenzial aus, das darin besteht, in ihrer Rationalität und mit der regulativen Idee der Aussagengeltung analysieren und beschreiben zu können, ,was tatsächlich Sache war. Im Gegensatz zu eher traditionellen Verfahren in der Begleitung von Praktika, in denen Studierende Erlebnisse und Erfahrungen berichten und dann subjektivistisch-meinend und in erster Linie lösungsorientiert daran angeschlossen wird, eröffnet die Orientierung an der Figur der Wissenschaftspraxis ein Spektrum an hochschuladäquaten Möglichkeiten: Angefangen bei der (technischen) Dokumentation der Vollzugswirklichkeit und der Transkription als vorbereitendem und gleichzeitig bisweilen bereits irritierendem Erkennen dessen, was tatsächlich stattgefunden hat, bis zu einer Analyse, die zunehmend kompetent zentrale Operationen wissenschaftlicher Methoden als Erkenntniswege für die dokumentierte Situation einsetzt, bietet die Hochschule ein Arsenal an Erkenntnis- und Reflexionsmöglichkeiten bezogen auf die berufliche Praxis.

So wertvoll die drei genannten Potenziale der Wissenschaft und ihrer Praxis für die berufliche Praxis sind, gilt es jedoch auch, die Limitationen im Verhältnis der beiden Praxen (wieder auf einer generellen Ebene) zueinander zu kennzeichnen. Die Differenz zwischen Sein und Sollen, also zwischen der Beschreibung eines sorgfältig erarbeiteten empirischen Befundes und der Frage nach den daraus abzuleitenden Konsequenzen, lässt sich systematisch nicht einebnen. Aus einem wissenschaftlichen Befund eine handlungspraktische, gar bildungspolitische Konsequenz auch nur als Empfehlung abzuleiten, bedeutet, den Modus der Deskription zu verlassen und unter Einbezug normativer Perspektiven 
Entscheidungen zu treffen, die sich aber nicht direkt aus dem Befund ableiten. Zumindest eine Pädagogische Hochschule kann damit nicht sinnvoll und aussichtsreich in der Absicht adressiert werden, stellvertretend für die berufliche Praxis deren Probleme zu lösen; gleichwohl kann sie beanspruchen, ihren genuinen Beitrag zur Produktion von Wissen unter Geltungsanspruch zu leisten.

Zusammenfassend lässt sich Folgendes festhalten: Abgesehen von Angeboten wie Hochschullernwerkstätten, Schüler*innenlaboren oder anderen Formen der Institutionalisierung studiumsbezogener Lehr-Lern-Arrangements unter Beteiligung von Schüler*innen sind die Berufspraktischen Studien der einzige Studienbereich, in dem sich Studierende zunehmend massgeblich an der beruflichen Praxis beteiligen. ${ }^{5}$ Sie tun das umso erfolgreicher, je stärker sie dabei auf das in den anderen Studienbereichen erworbene Wissen und Können zurückgreifen können. Zugleich sind die Berufspraktischen Studien selbst der Ort, an dem die subjektive und objektive Relevanz vorgängig erworbenen Wissens in besonderer Weise sichtbar werden kann und eine wissenschaftliche Bezugnahme auf vorgängige berufliche Praxis, wissenschaftspraktisch' eingeübt werden kann. Dementsprechend liegt der konzeptionelle Kern Berufspraktischer Studien darin, die von Studierenden mitzugestaltende berufliche Praxis vorgängig wissenschaftsgestützt und theoretisch informiert vorzubereiten, sie dann in Einlassung auf die situativen Bedingungen und unvermeidbaren Kontingenzen (mit) zu gestalten und sie nachträglich auf der Basis einer (ausschnitthaften, aber gezielten) Dokumentation methodengestützt zu analysieren.

\section{Fachlichkeit im Zyklus 1 und die Berufspraktischen Studien}

Auf der Basis der in Abschnitt 2 ausgeführten Überlegungen und konzeptionellen Rahmungen kann nun diskutiert werden, welchen Beitrag der Studienbereich Berufspraktischer Studien zu der im vorliegenden Band zentralen Frage der Konzeption von Fachlichkeit für den Zyklus 1 leisten kann. Die einfache Lösung, die Entwicklung einer elaborierten Fachlichkeit als Erwartung an die Studienbereiche der Fachdidaktik und der Fachwissenschaft zu delegieren und diese als Grundlage Berufspraktischer Studien vorauszusetzen, erweist sich empirisch und konzeptionell - auch angesichts der begrenzten Lehrveranstaltungsumfänge der einzelnen Fachwissenschaften und -didaktiken - als nicht tragfähig. Auch die z.B. in den Studiengängen zur Sekundarstufe geläufige Form von Fachpraktika ist

5 Selbst wenn diese berufliche Praxis mit Aufträgen oder Ähnlichem bisweilen didaktisch überformt ist, treffen Studierende nur dort auf Situationen und Bedingungen, die zumindest nah an den Anforderungen der beruflichen Praxis sind und die in den jeweiligen normativen Ordnungen vor Ort operieren. 
aus zwei Gründen für die ,Bildung der Generalistin bzw. des Generalisten ' im Zyklus 1 untauglich: Die fachbereichsspezifische Logik eines Fachpraktikums bricht sich zum einen an einer nicht oder nicht durchgängig fachlichen Unterrichtsrealität: Im Kindergarten findet kein Mathematikunterricht im engeren Sinne statt; insofern ist ein Praktikum nicht auf diese Perspektive zu reduzieren. ${ }^{6}$ Das zweite Argument ist organisatorischer Natur: Wenn jeder Fachbereich (an unserem Institut sechs) ein Fachpraktikum beanspruchte, wären die Praktika kurz und vermutlich wenig lernwirksam.

Der Erwerb einer tragfähigen Konzeption von Fachlichkeit bei Studierenden wird daher in den Berufspraktischen Studien und damit, im Angesicht von oft nicht explizit fachlich strukturierter beruflicher Praxis' an unserem Institut derzeit durch folgende zwei Prinzipien zu unterstützen versucht:

1. Studierende beteiligen sich (in über die Studienjahre zunehmend eigenständiger Weise und steigendem Umfang) an allen unterrichtlichen Aktivitäten ${ }^{7}$ im Praktikum. Studierende übernehmen also von Beginn an Unterrichtssequenzen, die sowohl fachbereichsverbindend oder fachbereichsvorbereitend (Thema „Winter" im Kindergarten) als auch explizit fachlich (Thema „Zehnerübergang" in Klasse 1) strukturiert sind.

2. Alle Fachbereiche beteiligen sich personell an den praktikumsbegleitenden Hochschulveranstaltungen und den Praktikumsbesuchen vor Ort. In dieser Beteiligung operieren sie konzeptionell als Könner*innen einer fachbereichsspezifisch orientierten Fach- und Wissenschaftspraxis, die damit systematisch nur einen (kleinen) Teil der beruflichen Realität expertenhaft erschliessen können.

Die beiden formulierten Gestaltungsprinzipien bergen unvermeidlich Chancen und Herausforderungen, die im Folgenden auf verschiedenen Ebenen diskutiert werden. Diese Darstellung dient auch als Grundlage für Perspektiven der auf Fachlichkeit bezogenen Weiterentwicklung der Konzeption Berufspraktischer Studien für die Zielstufe.

\section{Ebene 1: Konzeption}

Die konzeptionelle Chance der beiden Prinzipien liegt darin, dass Studierende mit allen (fachlich basierten) Anforderungen der beruflichen Praxis in Kontakt kommen und zugleich im fachbereichsspezifisch orientierten Studium das notwendige Struktur- und Orientierungswissen bezüglich der jeweils etablierten

6 Mit gewisser Regelmässigkeit führt dies im Praktikum zu Friktionen, weil dezidiert fachbereichsbezogene Ansprüche der Hochschulmitarbeitenden im Praktikum vor Ort als „nicht realisierbar“ eingeschätzt werden.

7 Sie beteiligen sich vor allem im zweiten Studienjahr im Langzeitpraktikum an Partnerschulen auch systematisch an ausserunterrichtlichen Aufgaben der Lehrer*innen, die hier aber nicht Gegenstand sind. 
Denk-, Arbeits- und Handlungsweisen erwerben. Die personelle Beteiligung aller Fachbereiche in den Berufspraktischen Studien stellt sicher, dass die Lehrenden in den anderen Studienbereichen dieselben sind, die auch Praktika begleiten. Eine Verselbstständigung bzw. Abkopplung der Berufspraktischen Studien wird damit vermieden. Eine Konzeption, in der die Bildungspotenziale unterrichtlicher Lernarrangements aus mehr als einer fachlichen Perspektive analytisch bzw. planend und gestaltend bearbeitet werden, elaborieren Künzli David und de Sterke im vorliegenden Band.

Herausforderungen auf konzeptioneller Ebene bestehen darin, die institutionellen Orte im Studiengang zu schaffen, in denen sich Studierende mit der für die Stufe typischen ,Doppelgestalt von Fachlichkeit' auseinandersetzen: einerseits als fachbereichsübergreifende Auseinandersetzungen, in denen es die jeweiligen fachlichen Potenziale gezielt zu identifizieren, bewusst zu planen und (fach)didaktisch angemessen zu realisieren gilt, andererseits als gezielt fachliche Lernanlässe, die entweder in fachlich orientierten Lernsequenzen gestaltet oder in offenen Lernsequenzen wahrgenommen und mit der (fachlich) adäquaten Lernunterstützung gefördert werden. Diese Orte sind idealerweise in ihrer Funktion eindeutig bestimmt; ihre Bestimmung wird im zuständigen Institut geteilt und die Formen der Auseinandersetzung in ihnen sind explizit und wiederkehrend. ${ }^{8}$

\section{Ebene 2: Mitarbeitende der Hochschule}

Die systematische Beteiligung von Mitarbeitenden aller Fachbereiche beinhaltet die wesentliche Chance, im Kontakt mit der Rationalität der beruflichen Praxis zu bleiben. Die deskriptiven Deutungsangebote und die präskriptiven pädagogisch-didaktischen Programme (vgl. Idel/Schütz 2016; Leonhard/Lüthi 2017), die an der Hochschule in diversen Modi Gegenstand der Lehre sind, erfahren im Kontakt mit der beruflichen Praxis potenziell eine empirische Prüfung der systematischen und situativen Deutungsmächtigkeit. Arbeitspsychologisch ermöglicht das Modell systematischer Beteiligung der Mitarbeitenden aller Fachbereiche in den Berufspraktischen Studien ausserordentlich vielfältige Arbeitsverhältnisse. In Fachwissenschaft und -didaktik zu lehren, gegebenenfalls zu forschen und sich an der Begleitung Berufspraktischer Studien zu beteiligen, stellt potenziell eine Bereicherung dar; es kann zudem ein Regulativ sein, die eigenen Ansprüche mit der Empirie ihrer Realisierbarkeit in Beziehung zu setzen. Mit der Vielfalt an Tätigkeiten gehen jedoch auch besonders hohe Qualifikationsanforderungen einher, die entweder die Gewinnung derart gut qualifizierter Mitarbeitender zur Heraus-

8 Auf die Herausforderung, nicht nur Studierende in diesen institutionellen Räumen für die konzeptionellen Perspektiven zu gewinnen, sondern auch zumindest die erfahrenen Lehrer*innen, die Studierende in den Praktika begleiten, sei an dieser Stelle nur verwiesen. Diese Aufgabe ist angesichts oft schwacher institutioneller Einbindung dieses Personenkreises besonders anspruchsvoll und sicher nur auf Ebene eines Instituts, nicht aber in den Berufspraktischen Studien allein zu lösen. 
forderung werden lässt oder spezifische Qualifizierungen ,on the job` erforderlich macht.

Mitarbeitende Pädagogischer Hochschulen, die sowohl in einem Fachbereich als auch in den Berufspraktischen Studien in der Lehre beteiligt sind, benötigen ein mehrfaches ,Kompetenzprofil', das im Folgenden etwas detaillierter ausgeführt wird. In der Schweiz wird derzeit an Fachhochschulen und Pädagogischen Hochschulen die Figur des sogenannten „Doppelten Kompetenzprofils“ diskutiert ${ }^{9}$ und mit Forschungs- und Entwicklungsmitteln lanciert. Es läuft aufgrund seiner institutionenpolitischen Prominenz Gefahr, zum Dogma zu werden, das konkrete Einstellungsvoraussetzungen ohne die entsprechende aufgabenbezogene Differenzierung und Nuancierung vorschreibt. Der damit verbundene Anspruch resultiert aus der Annahme, dass Lehrende an den genannten Hochschultypen sowohl über eigene einschlägige berufliche Erfahrungen (,Lehrdiplom', ,Zielstufenbezug' nach Bachelorabschluss) als auch über eine wissenschaftliche Qualifikation verfügen mögen. Ratifiziert man diese Überlegung, ${ }^{10}$ wird das Anforderungsprofil an Mitarbeitende, die zusätzlich zur Lehre in ihrem Fachbereich die Berufspraktischen Studien mitgestalten, nochmals anspruchsvoller, insbesondere dann, wenn eine Konzeption vorliegt, die den Studienbereich als akademischen konzipiert: Wo die Begleitung Berufspraktischer Studien mehr ist als Erfahrungsaustausch, kollegiale Fallberatung und (fach)didaktische Planungshinweise und theoretisch fundierte Planungen ebenso bedeutsam sind wie daten- und methodengestützte Analysen von Unterricht mit einem Anspruch an Aussagengeltung, wird aus dem „Doppelten Kompetenzprofil“ fast ein dreifaches. Als Voraussetzung für die Beteiligung an den Berufspraktischen Studien ist dies nur in den seltensten Fällen erwartbar und meist nur als längerfristiger Prozess partizipativer Kompetenzentwicklung für dieses breite Anforderungsprofil zu realisieren.

\section{Ebene 3: Mitarbeitende der beruflichen Praxis}

Mit Praxislehrpersonen, die in den Berufspraktischen Studien den Auftrag haben, Studierende in ihren Praktika zu begleiten, ein Modell der Bearbeitung der beruflichen Anforderungen zu sein und ihnen intensive berufsfeldbezogene Erfahrungen ebenso zu ermöglichen wie diese mit ihnen kriterienorientiert zu diskutieren, hat die Hochschule einen in mehrfacher Hinsicht ,authentischen Berufspraxisbe-

9 Vgl. z.B. https://www.swissuniversities.ch/themen/nachwuchsfoerderung/p-11-doppeltes-kompetenzprofil.

10 Eine solche Ratifizierung ist aus der Sicht des Autors keineswegs zwingend. Vielmehr ist zu befürchten, dass es sich bei dieser Figur um einen konzeptionellen Kollateralschaden des, TheoriePraxis-Narrativs' handelt, der die Institutionen in die Gefahr einer systematischen Selbstüberforderung bringt, anstatt sie - wie im vorliegenden Beitrag vorgeschlagen - auf die fokussierte Auseinandersetzung mit der eigenen und hochschuladäquaten Praxis zu verpflichten, deren spezifische Leistungen für die jeweilige berufliche Praxis eindeutig ausweisbar und zugleich eindeutig limitiert sind. 
zug' institutionalisiert. In den Praktika - selbst wenn sie in Teilen didaktisiert und als Ausbildungssituation auch geschützt sind - erleben die Studierende eine berufliche Praxis, die den theoretischen Konzepten und den Programmatiken der Hochschule immer schon vorgängig war und trotz einer inhaltlich-konzeptionellen Vorstrukturierung der Praktika durch Artefakte wie ,Leitfäden' oder Ähnliches durchaus eigensinnig damit umgeht. So werden Anforderungen berufsfeldspezifisch rekontextualisiert oder bisweilen weitgehend ignoriert, weil z.B. die Überzeugung, dass man im eigenverantwortlichen Tun am meisten lerne oder dass man in einem Abschlusspraktikum auch unter Beweis stellen müsse, dass man den vollen Anforderungen des Berufs gewachsen sei, übermächtig ist. Nicht selten haben dort z.B. unter der Leitidee von, Ganzheitlichkeit' dezidiert fachliche Perspektiven eine andere Priorität oder werden gar zum Stein des Anstosses, weil z.B. fachdidaktische Anforderungen, die auch in Musik eine Progression an unterscheidbaren Fähigkeiten beanspruchen, zurückgewiesen werden, weil Musik im Sinne gemeinsamen Singens oder rhythmischer Verse vorwiegend gemeinschaftsstiftend und übergangsstrukturierend und damit eher funktional und nicht als eigenständiger Bildungsbereich angesehen wird. In solchen Situationen sind seitens der Hochschulmitarbeitenden gute Argumente, die Möglichkeit zur praktischen Modellierung der Ansprüche, legitimatorisch auch der Verweis darauf, ,selbst unterrichtet zu haben', und das notwendige diplomatische Geschick erforderlich. Sind diese Anforderungen erfüllt und ist die Einlassung aller Beteiligten gegeben, sind fruchtbare Momente einer wechselseitigen Bezugnahme, Anerkennung und gemeinsamer Lernprozesse möglich. Die Wahrscheinlichkeit dafür steigt erheblich, wenn es gelingt, längerfristige Zusammenarbeitsbeziehungen zu installieren, welche die Bildung einer Vertrauensbeziehung ermöglichen. In Langzeitpraktika, die an speziellen Partnerschulen stattfinden, sind derartige Prozesse wechselseitiger Anerkennung empirisch durchaus beobachtbar; sie erfordern aber ebenso hohe Kompetenz wie hohen Einsatz der Beteiligten.

\section{Ebene 4: Organisatorische Rahmenbedingungen}

Unzweifelhaft spielen organisatorische Rahmenbedingungen eine zentrale Rolle bei der Frage nach der Realisierbarkeit ambitionierter Konzeptionen, und zwar insbesondere dann, wenn sie mehr als eine einzelne Organisationseinheiten betreffen und ein Commitment in zentralen Fragen z.B. auf Institutsebene angestrebt wird. Die Spezifik der jeweiligen Rahmenbedingungen macht es jedoch an dieser Stelle wenig sinnvoll, detaillierte Erwägungen vorzunehmen.

Für die nachfolgenden Überlegungen einer Weiterentwicklung ist es jedoch erforderlich, die Konsequenzen aus den oben dargestellten Gestaltungsprinzipien festzuhalten: 
- Es ist ungewiss, welche (fach)didaktische Begleitung die Studierenden im Laufe ihres Studiums in den Berufspraktischen Studien erfahren. Auch im günstigen Fall ist nur ein Teil der Fachbereiche beteiligt.

- Der hohe Anspruch der Konzeption und die zeitliche Inanspruchnahme der Mitarbeitenden in den Zwischensemestern für die Berufspraktischen Studien schmälert die Attraktivität, sodass die Beteiligung im Mittel eher ,verhalten ist. Diese Zurückhaltung verringert die Wahrscheinlichkeit der wirksamen Einflussnahme auf eine elaborierte Fachlichkeit.

- Die konzeptionelle Argumentation bezüglich einer methodisch kontrollierten Analyse als genuin hochschuladäquater Form der Auseinandersetzung mit Situationen und Produkten der beruflichen Praxis präformiert eine methodologische Orientierung auf sozialwissenschaftliche, qualitative bzw. qualitativ-rekonstruktive Methoden, die eine sozialwissenschaftliche Kasuistik begründen (vgl. dazu Beck/Helsper/Heuer/Stelmaszyk/Ullrich 2000; Hummrich/Hebenstreit/Hinrichsen/Meier 2016; Idel/Schütz 2016; Ohlhaver/Wernet 1999; Pieper/Frei/Hauenschild/Schmidt-Thieme 2014; Reh/Rabenstein 2005). Derartige Methoden sind bisher nicht in allen Fachbereichen selbstverständlicher Teil des Studiums. Sie erfordern in den Berufspraktischen Studien Erfahrung und die Ausprägung einer spezifischen Könner*innenschaft als Methodenpraxis und ihrer ,hochschuldidaktischen 'Vermittlung, die in diesem Kontext eine „Wissenschaftsdidaktik“ (vgl. dazu Schaller 1970; Schmohl 2019) der Teilnahme an ebendieser Praxis ist. Es braucht angesichts der studentischen Alltagserwartungen, die eher auf Austausch und Problemlösung als auf vertieftes Verstehen zielen, ein ebenso überzeugtes wie überzeugendes Auftreten und die Fähigkeit, trotz (nachvollziehbarer) Skepsis gegenüber den Mühen mit der methodisch geleiteten Analyse die Spannung bis zu dem Punkt aufrechtzuerhalten, an dem zumindest für einen Teil der Studierenden der Gewinn an Einsicht in den Sinn dieser Bemühungen erlebbar wird.

- Es gilt auch festzuhalten, dass die Ausarbeitung und die Adaption geeigneter Methoden zur Analyse unterrichtlicher Situationen in den Berufspraktischen Studien erst am Anfang stehen und insbesondere in Bezug auf fachdidaktische Fragestellungen noch ein gewisses Desiderat darstellen (vgl. Leonhard et al. 2021).

- Die systematischen Beziehungen zwischen den Mitarbeitenden der Hochschulen und den mitarbeitenden Praxislehrpersonen an den Schulen sind geeignet, um eine etablierte Zusammenarbeit stabil fortzusetzen. Für die Steigerung des wechselseitigen Verständnisses fehlen regelmässige Formate der fachlich-inhaltlichen und ausbildungsbezogenen Zusammenarbeit.

Insgesamt lässt sich feststellen, dass die bisherige Konzeption Berufspraktischer Studien am Institut Kindergarten-/Unterstufe das Potenzial enthält, fachliche 
Perspektiven wissenschafts- und berufspraktisch zur Geltung zu bringen. Es ist jedoch auch zu konstatieren, dass die Potenziale derzeit noch nicht umfassend realisiert werden. Insofern stellt sich die Frage nach einer Weiterentwicklung der Konzeption.

\section{Entwicklungsperspektiven}

Im letzten Abschnitt des vorliegenden Beitrag wird vor dem Hintergrund der vorangegangenen Ausführungen (in ersten Ansätzen) die Idee ausgearbeitet, das Konzept des „Transversalen Unterrichtens“ (vgl. Künzli David und de Sterke in diesem Band) als zentralen Bezug zu wählen und dessen Spezifik, Fachlichkeit in Zyklus 1 begrifflich präzise zu fassen und unterrichtlich wirksam werden zu lassen, in den Berufspraktischen Studien stärker zu integrieren. Zweifelsohne leisten auch der ganze Band und die darin enthaltenen Darlegungen der fachbereichsspezifischen Perspektivierungen einen wesentlichen Beitrag zu einer ,geklärten Vielfalt', die gegebenenfalls in der Figur der „Modi der Weltbegegnung" (Dressler 2013, 187) eine geeignete bildungstheoretische Rahmung erfährt. Diese vermag auch für die Studierenden Zusammenhänge zu stiften sowie Differenzen erkennbar werden zu lassen.

Die Orientierung am Konzept Transversalen Unterrichtens lässt sich auf verschiedenen Ebenen denken. Im Folgenden werden zunächst Voraussetzungen skizziert, um daran anschliessend Vorschläge auf der konkret operativen Ebene zu prüfen und zuletzt auch hochschulorganisatorisch anspruchsvolle Formen in ihren Potenzialen und Passungsmomenten zu den konzeptionellen Leitideen der Berufspraktischen Studien anzudenken.

\section{Voraussetzungen}

Fasst man die Zielperspektive und den Anspruch des Transversalen Unterrichtens darin, dass Lehrer*innen des Zyklus 1 in der Lage sind, thematisch-lebensweltliche Unterrichtsarrangements vor dem Hintergrund differenzierter und vielfältiger fachlicher Perspektiven (statt der diffusen Programmatik von ,Ganzheitlichkeit') zu planen, zu gestalten und situativ zu begleiten, so lässt sich als elementare Voraussetzung beschreiben, dass diese Grundüberlegung einen institutionellen Ort erfordert, dem alle Studierenden des Studiengangs früh und zugleich mehrfach begegnen, um das Verständnis für die Ansprüche sukzessive zu entwickeln. Insofern spricht einiges dafür, die konzeptionelle Abstimmung zu intensivieren. Als weitere Voraussetzung kann sicher ein bei Mitarbeitenden möglichst aller Fachbereiche fundiert etabliertes Verständnis des Konzepts des Transversalen Unterrichtens gekennzeichnet werden. 


\section{Konkret-operative Ideen}

Ein Ziel der ersten Phase der Berufspraktischen Studien besteht in der gezielten Beobachtung der beruflichen Praxis vor allem der Praxislehrpersonen. Bereits jetzt existiert eine Reihe von Beobachtungs- bzw. Erkundungsaufträgen, die im Modus der Distanz während der Hospitationen im ersten Semester bearbeitet werden. Ein verbindlicher Beobachtungsauftrag, der Transversalität als Unterrichtsprinzip erkennbar werden lässt und in den beobachteten Unterrichtsarrangements realisierte wie in situ nicht realisierte fachliche Perspektivierungen untersucht, könnte organisatorisch unaufwändig zur Sensibilisierung der Studierenden und zu ersten Erkundungen der differenten Logiken des Studiums und der beruflichen Praxis beitragen. „Welche fachlichen Zielperspektiven berichten Praxislehrpersonen in Bezug auf die Gestaltung einer an die Lebenswelt der Kinder anknüpfenden Themenstellung?“ oder „Beobachten Sie, welche Hinweise eine Lehrperson den Kindern in Phasen offener Lernarrangements gibt und versuchen Sie, die darin sichtbar werdenden fachlichen Perspektiven zu identifizieren" könnten hier geeignete Impulse sein. Der gemeinsame Blick auf ein entsprechendes empirisches Datum wie einen Videoausschnitt oder ein Transkript schärft die Wahrnehmung und etabliert sukzessive die erforderliche Differenzierungsfähigkeit.

\section{Weiterführende Überlegungen}

Konzeptionell attraktiv, wenngleich organisatorisch deutlich anspruchsvoller sind Ideen, die darauf abzielen, die differenten Fachperspektiven in den Berufspraktischen Studien anlassbezogen personell zu vertreten und expertenhaft modellieren zu können. Anlässlich einer zielstufentypischen Themenstellung wie „Winter“ Planungsprozesse mit Studierenden zu gestalten, in denen bewusst verschiedene „Modi der Welterschliessung“ (Dressler 2013, 187) gedankenexperimentell geprüft werden, scheint konzeptionell aussichtsreich, um den Gedanken multipler fachlicher Perspektiven bei Studierenden zu etablieren. Mit fachlichen Expertinnen und Experten Unterrichtsarrangements zu konzipieren, in denen Schüler*innen die Möglichkeiten sowohl zur Teilnahme an spezifischen kulturellen Praktiken als auch zur beobachtenden Distanzierung haben, die eine Voraussetzung für eine zunehmende Differenzierung von Bezugnahmen auf die Welt darstellt (vgl. Dressler 2013, 195f.), kann absehbar dazu beitragen, das Konzept der Transversalität als Spezifikum des Unterrichts im Zyklus 1 zu etablieren und damit einen Beitrag zu einem eigenständigen beruflichen Selbstkonzept von Lehrpersonen dieser Stufe zu leisten. Analog lassen sich konzeptionelle Ideen zur nachträglichen Analyse empirischer Daten aus dem Unterricht entwickeln, die - expertenhaft modelliert - fachbereichsspezifische Denk-, Arbeits- und Handlungsweisen gerade auch in ,nicht fachlich konstituiertem Unterricht ${ }^{`}$ unterscheiden hilft und damit einen Anspruch sichtbar macht, der auch und gerade auf dieser Stufe geltend zu machen ist. Abgesehen von der Wirkung für die Studierenden sind derartige kooperative 
Arrangements aussichtsreiche Wege der Verständigung unter den Mitarbeitenden der Hochschule im Sinne interdisziplinärer Zusammenarbeit. Identisches gilt selbstverständlich für die ebenfalls hochplausible Idee, neben diversen fachlichen Perspektiven auch Vertreter*innen der beruflichen Praxis zur Partizipation einzuladen.

Derartige konzeptionelle Überlegungen geraten bisweilen erheblich unter Druck, wenn sich Fragen der Organisier- und Finanzierbarkeit stellen. Da diese Fragen zumeist nur sehr hochschulspezifisch zu beantworten sind, endet der Beitrag mit der Feststellung, dass eine fortgesetzte institutsinterne Verständigung auf konzeptioneller Ebene ebenso aussichtsreich erscheint wie die Anknüpfung an das Konzept von Transversalität im Zyklus 1.

\section{Literatur}

Beck, C., Helsper, W., Heuer, B., Stelmaszyk, B. \& Ullrich, H. (2000): Fallarbeit in der universitären LehrerInnenbildung Professionalisierung durch fallrekonstruktive Seminare? Eine Evaluation. Wiesbaden: VS Verlag für Sozialwissenschaften.

Bonnet, A. (2019): Die Rolle von Fachlichkeit für die Professionalität und Professionalisierung von Lehrer_innen - theoretische Überlegungen und empirische Befunde. In: Zeitschrift für interpretative Schul- und Unterrichtsforschung, 8. Jg., 164-177.

Bonnet, A. (2020): Das Verhältnis fachlicher und generischer Aspekte der Professionalität und Professionalisierung von Lehrerinnen und Lehrern - Erkenntnisse der strukturtheoretischen und berufsbiographischen Forschung. In: U. Hericks, M. Keller-Schneider, W. Meseth \& A. Rauschenberg (Hrsg.), Fachliche Bildung und Professionalisierung von Lehrerinnen und Lehrern. Bad Heilbrunn: Klinkhardt, 27-48.

Dressler, B. (2013): Fachdidaktik und die Lesbarkeit der Welt. Ein Vorschlag für ein bildungstheoretisches Rahmenkonzept der Fachdidaktiken. In: K. Müller-Roselius (Hrsg.), Bildung - empirischer Zugang und theoretischer Widerstreit. Opladen: Budrich, 183-202.

Hackl, B. (2014): Praxis der Theorie und Theorie der Praxis. Die Rekonstruktion von Unterrichtsszenen als Medium der Vermittlung von Wissenschaft und didaktischem Handeln. In: I. Schrittesser, I. Malmberg, R. Mateus-Berr \& M. Steger (Hrsg.), Zauberformel Praxis. Zu den Möglichkeiten und Grenzen von Praxiserfahrungen in der Lehrerbildung. Wien: new academic press, 51-68.

Hillebrandt, F. (2014): Soziologische Praxistheorien: Eine Einführung. Wiesbaden: Springer.

Hillebrandt, F. (2015): Was ist der Gegenstand einer Soziologie der Praxis? In: F. Schäfer, A. Daniel \& F. Hillebrandt (Hrsg.), Methoden einer Soziologie der Praxis. Bielefeld: transcript, 15-36.

Hummrich, M., Hebenstreit, A., Hinrichsen, M. \& Meier, M. (2016): Was ist der Fall? Kasuistik und das Verstehen pädagogischen Handelns. Wiesbaden: Springer VS.

Idel, T.-S. \& Schütz, A. (2016): Praxistheoretische Kasuistik im Lehramtsstudium. Wie man mit Studierenden über pädagogische Normen ins Gespräch kommen kann. In: M. Hummrich, A. Hebenstreit, M. Hinrichsen \& M. Meier (Hrsg.), Was ist der Fall? Kasuistik und das Verstehen pädagogischen Handelns. Wiesbaden: Springer VS, 63-80.

Klafki, W. \& Stöcker, H. (1976): Innere Differenzierung des Unterrichts. In: Zeitschrift für Pädagogik, 22. Jg. (H. 4), 497-523.

Košinár, J., Leineweber, S. \& Schmid, E. (Hrsg.) (2016): Professionalisierungsprozesse angehender Lehrpersonen in den berufspraktischen Studien. Münster: Waxmann.

Leonhard, T. (2018): Zwischen Baum und Borke? Zur Eigenlogik schul- bzw. berufspraktischer Studien. In: J. Böhme, C. Cramer \& C. Bressler (Hrsg.), Erziehungswissenschaft und Lehrerbildung 
im Widerstreit!? Verhältnisbestimmungen, Herausforderungen und Perspektiven. Bad Heilbrunn: Klinkhardt, 211-222.

Leonhard, T. (2020): Verlängerte Praxisphasen und das ,Theorie-Praxis-Problem ' - Perspektiven der Professionalisierung durch eine gewandelte Rahmung. In: K. Rheinländer \& D. Scholl (Hrsg.), Verlängerte Praxisphasen in der Lehrer*innenbildung. Konzeptionelle und empirische Aspekte der Relationierung von Theorie und Praxis. Bad Heilbrunn: Klinkhardt, 39-57.

Leonhard, T. (2021): Der Fall in den Schul- und Berufspraktischen Studien. In: D. Wittek, T. Rabe \& M. Ritter (Hrsg.), Kasuistik in Forschung und Lehre - erziehungswissenschaftliche und fachdidaktische Ordnungsversuche. Bad Heilbrunn: Klinkhardt, im Erscheinen.

Leonhard, T., Herzmann, P. \& Košinár, J. (Hrsg.). (2021): „Grau, theurer Freund, ist alle Theorie“?Theorien und Erkenntniswege Schul- und Berufspraktischer Studien. Münster: Waxmann.

Leonhard, T. \& Lüthi, K. (2017): Pädagogischen Programmen auf der Spur: Bildungspotenziale an der Differenz zwischen pädagogischem Anspruch und seiner Realisierung. In: T. Burger \& M. Nicole (Hrsg.), Empirische Forschung im Kontext Schule. Wiesbaden: Springer VS, 67-83.

Meister, N. \& Hericks, U. (2021): Reflektierte Fachlichkeit und doppeltes Praxisverständnis. Studienkonzeptionelle Grundlagen und ihre Umsetzung. In: T. Leonhard, P. Herzmann \& J. Košinár (Hrsg.), Grau, theurer Freund, ist alle Theorie? Theorien und Erkenntniswege Schul- und Berufspraktischer Studien. Münster: Waxmann, im Erscheinen.

Neuweg, G. H. (2011): Distanz und Einlassung. Skeptische Anmerkungen zum Ideal einer Theorie-Praxis-Integration in der Lehrerbildung. In: Erziehungswissenschaft, 22. Jg. (H. 43), 33-45.

Ohlhaver, F., \& Wernet, A. (1999): Schulforschung - Fallanalyse - Lehrerbildung. Diskussionen am Fall. Opladen: Leske + Budrich.

Pieper, I., Frei, P., Hauenschild, K. \& Schmidt-Thieme, B. (2014): Was der Fall ist. Beiträge zur Fallarbeit in Bildungsforschung, Lehramtsstudium, Beruf und Ausbildung. Wiesbaden: Springer.

Reckwitz, A. (2003): Grundelemente einer Theorie sozialer Praktiken: Eine sozialtheoretische Perspektive. In: Zeitschrift für Soziologie, 32. Jg. (H. 4), 282-301.

Reh, S., \& Rabenstein, K. (2005): „Fälle“ in der Lehrerausbildung. Schwierigkeiten und Grenzen ihres Einsatzes. In: Journal für Lehrerinnen- und Lehrerbildung, 5. Jg. (H. 4), 47-54.

Schäfer, H. (2016): Praxistheorie: Ein soziologisches Forschungsprogramm. Bielefeld: transcript.

Schaller, K. (1970): Fragestellungen der gegenwärtigen Diskussion um Hochschuldidaktik. In: K. Schaller (Hrsg.), Wissenschaft und Lehre. Hochschuldidaktische Vorschläge und Versuche. Heidelberg: Quelle \& Meyer, 5-17.

Schmidt, R. (2012): Soziologie der Praktiken: Konzeptionelle Studien und empirische Analysen. Berlin: Suhrkamp.

Schmohl, T. (2019): Wie weiter in der Hochschullehrerbildung? In: Beiträge zur Lehrerinnen- und Lehrerbildung, 37. Jg. (H. 1), 110-125.

Schneider, J., \& Cramer, C. (2020): Relationierung von Theorie und Praxis: Was bedeutet dieses Konzept für die Begleitung von Praktika in der Lehrerinnen- und Lehrerbildung? In: K. Rheinländer \& D. Scholl (Hrsg.), Verlängerte Praxisphasen in der Lehrer*innenbildung. Konzeptionelle und empirische Aspekte der Relationierung von Theorie und Praxis. Bad Heilbrunn: Klinkhardt, 23-38.

Schrittesser, I. \& Hofer, M. (2012): Lehrerbildung als kulturelle Praxis? Wie Pierre Bourdieus Habitusbegriff die Kulturen der Lehrerbildung und der Schulpraxis einander näher bringen könnte ... In: C. Kraler, H. Schnabel-Schüle, M. Schratz \& B. Weyand (Hrsg.), Kulturen der Lehrerbildung: Professionalisierung eines Berufsstands im Wandel. Münster: Waxmann, 141-154.

Volpert, W. (2003): Wie wir handeln - was wir können. Ein Disput als Einführung in die Handlungspsychologie. Sottrum: Artefact Verlag Weber.

Wenzl, T., Wernet, A. \& Kollmer, I. (2018): Praxisparolen. Dekonstruktionen zum Praxiswunsch von Lehramtsstudierenden. Wiesbaden: Springer VS. 